\title{
Performance Improvement of Space Shift Keying MIMO Systems with Orthogonal Codebook-Based Phase-Rotation Precoding
}

\author{
Mohammed Al-Ansi, ${ }^{1}$ Syed Alwee Aljunid, ${ }^{1}$ and Essam Sourour ${ }^{2}$ \\ ${ }^{1}$ School of Computer and Communication Engineering, Universiti Malaysia Perlis (UniMAP), Perlis, Malaysia \\ ${ }^{2}$ Department of Electrical Engineering, College of Engineering at Wadi Aldawaser, Prince Sattam Bin Abdulaziz University, \\ Wadi Aldawaser 11991, Saudi Arabia \\ Correspondence should be addressed to Essam Sourour; sourour@ieee.org
}

Received 27 May 2017; Revised 27 September 2017; Accepted 2 October 2017; Published 9 November 2017

Academic Editor: Xianfu Lei

\begin{abstract}
Copyright (C) 2017 Mohammed Al-Ansi et al. This is an open access article distributed under the Creative Commons Attribution License, which permits unrestricted use, distribution, and reproduction in any medium, provided the original work is properly cited.
\end{abstract}

\begin{abstract}
This paper considers codebook-based precoding for Space Shift Keying (SSK) modulation MIMO system. Codebook-based precoding avoids the necessity for full knowledge of Channel State Information (CSI) at the transmitter and alleviates the complexity of generating a CSI-optimized precoder. The receiver selects the codeword that maximizes the Minimum Euclidean Distance (MED) of the received constellation and feeds back its index to the transmitter. In this paper, we first develop a new accurate closed-form Bit Error Rate (BER) for SSK without precoding. Then, we investigate several phase-rotation codebooks with quantized set of phases and systematic structure. Namely, we investigate the Full-Combination, Walsh-Hadamard, Quasi-Orthogonal Sequences, and Orthogonal Array Testing codebooks. In addition, since the size of the Full-Combination codebook may be large, we develop an iterative search method for fast selection of its best codeword. The proposed codebooks significantly improve the BER performance in Rayleigh and Nakagami fading channels, even at high spatial correlation among transmit antennas and CSI estimation error. Moreover, we show that only four phases $\{+1,+j,-1,-j\}$ are sufficient and further phase granularity does yield significant gain. This avoids hardware multiplication during searching the codebook and applying the codeword.
\end{abstract}

\section{Introduction}

Spatial Modulation (SM) is a novel technique to achieve low complexity and energy efficient Multiple Input Multiple Output (MIMO) systems [1]. In SM, part of the user data bits is conveyed through the index of a single transmit antenna (TA), while the second part is conveyed through classic selection of an Amplitude and Phase Modulation symbol [1, 2]. One special form of SM-MIMO is the Space Shift Keying (SSK) proposed in [3] to tradeoff receiver complexity for data rate. In SSK, the data bits are encoded only in the index of TA, which transmits an unmodulated data such as +1 symbol. When the number of TAs is not sufficient, Generalized SSK and Generalized SM are introduced, respectively, in [4] and [5] to increase the spectral efficiency by activating multiple TAs and availing larger TA combinations and indexes.

In comparison to the conventional MIMO schemes, SM has potential advantages. Notably among them are the avoidance of interchannel interference and relaxation of the need for interantenna synchronization, due to the transmission from a single antenna at each time instant. The complexity of the transceiver is also reduced and the energy efficiency is enhanced since only one Radio Frequency (RF) chain is required. Furthermore, the configurations related to the number of transmit and receive antennas are flexible in contrast to the restrictions applied in MIMO spatial multiplexing $[1-3,6]$. Initial research was dedicated to improve the SM and SSK systems performance through designing low complexity receiver algorithms that minimize the Bit Error Rate (BER) [7-9]. However, Transmit Precoding (TPC) as a preprocessing technique on the transmitter side, with possible feedback from the receiver side, has lately shown remarkable performance gains [10, 11].

TPC assumes downlink Channel State Information (CSI) is known at least at the receiver, and a common precoding codeword is employed by the transmitter and the receiver. In the transmitter side, the codeword is used to scale the 
TAs. In the receiver side, it is used in Maximum Likelihood (ML) detection of the TA index and the transmitted symbol [8]. In most research, the design objective of TPC codeword is maximizing the Minimum Euclidean Distance (MED) between pairs of received symbols, which is the dominant factor affecting the BER performance [3]. Finding the codeword that fulfills MED maximization has been the subject of considerable research, which can be categorized into three main approaches as described below.

The first approach, which is a noncodebook approach, captured the most attention in the TPC research. This approach develops algorithms to find the TPC codeword that realizes a design objective for the given CSI [1220]. This algorithm should be used in the transmitter and receiver to yield the same codeword, and it requires full knowledge of the CSI at both sides. The most widely used design objective is the maximization of the MED subject to fixed transmit power. This is sometimes termed as the Maximum Minimum Distance (MMD), and it is used as the first method in $[12,13]$. Another utilized design objective is the transmit power minimization subject to Guaranteed Euclidean distance (GED), which is utilized as the second method in $[12,13]$. Other design objectives include Signalto-Noise Ratio (SNR) maximization [14], direct BER minimization [15], and phase alignment [16]. In [12-14, 17, 18], the designed codeword involves amplitude scaling, while, in $[15,16]$, it is based on phase rotation only. Therefore, this approach yields a codeword matching the instantaneous channel, leading to a significant reduction in BER. It is more practical in Time Division Duplex (TDD) systems where the uplink and downlink channels are the same. However, there are some limitations to this approach. The solution for the codeword is typically iterative. Depending on the number of transmit and receive antennas the complexity of the algorithm may be an issue. Also, the required number of iterations is variable and depends on the instantaneous CSI and SNR, leading to a nonfixed processing time. Moreover, when the RF chain specifications and processing power of the receiver and transmitter are different, the reciprocity may be an issue. Besides, huge overhead is required to obtain full CSI through feedback with infinite rate, particularly in the Frequency Division Duplex (FDD) systems [21]. To solve this latter problem, the research in [19] suggests quantizing the channel phase angles at the receiver, and feeding them back to the transmitter with limited number of bits, to be used for phase compensation at the transmitter. Also, [20] proposes a simplified power allocation method. The receiver uses the CSI to update the power allocation for only two antennas that are associated with MED and feeds back only their indices and updated power allocation using a limited number of bits.

The second approach is the CSI-independent codebookbased approach $[22,23]$. This approach is proposed to overcome the aforementioned limitations of the first approach through utilizing a codebook with a limited predefined number of codewords known by the transmitter and receiver. Given the CSI, the receiver selects the codeword that maximizes the MED and feeds back the index of the selected codeword to the transmitter. This feedback can be considered as a limited knowledge of CSI at the transmitter. The second method of codebook construction proposed in [22] follows this approach. The codebook is the DFT matrix, where the codebook size should be less than or equal to the number of TAs. Interestingly, a modified DFT matrix is also utilized in [24] as a precoding matrix for a special form of Generalized SSK system, under spatially correlated channels. The precoding matrix is constructed by multiplying a complex random unitary matrix by the DFT matrix. It is worthwhile to mention that [24] does not assume any feedback from the receiver to the transmitter. The precoding codeword is selected by the bit sequence to be transmitted, rather than the instantaneous CSI. However, the utilization of the CSI-independent precoder falls into the second approach. In [23], a codebook was constructed of randomly generated matrix of complex Gaussian random variables with zero mean and unit variance. Significant performance gain has been shown in [22-24] with CSI-independent codebooks. In [25], the authors of this paper presented two small-size codebooks that are subsets of all possible combinations of codewords (denoted as FullCombination codebook). The first codebook is the Factorized Full-Combinations codebook, which is based on restructuring the Full-Combination codebook for faster codeword search. The second codebook is the Statistically Filtered FullCombinations codebook, which is based on selecting the codewords which are most frequently used. While these two codebooks provide good performance, they do not have a predefined structure. The CSI-independent codebook-based approach is suitable for FDD systems that are widely used in $3 \mathrm{G}$ and $4 \mathrm{G}$ systems, where the uplink and downlink channels are not reciprocal. Also, these codebooks require only a limited knowledge of CSI at the transmitter. However, the drawback of this approach is that the selected codeword does not match the channel exactly. Hence, the MED is not globally maximized, which may lead to performance loss compared to the first approach.

The third approach is a hybrid approach [26], where a codebook is designed to match the channel on the average. The first method of codebook construction proposed in [22], as well as the method in [27] by the same authors, follows this approach. In [22], the codebook is constructed by generating a large set of random codewords. Then, this set is filtered to select the best codebook with the desired number of codewords. The selection criterion is the maximization of the statistical expectation of the MED. In [27], the codebook is generated iteratively using the concept of the Lloyd algorithm [28] and the nearest neighborhood and centroid criteria. A training set of 6000 random channel realizations are used as the training set for codebook construction. In [22, 27], once the codebook is constructed, codeword is selected based on maximizing the MED and the index is fed back from the receiver to the transmitter. In [24], in addition to the DFT-based precoder mentioned in the second approach, the authors also propose two codebook construction methods based on zero-forcing and matched filter of the transmit correlation matrix. Hence, these codebooks fall in the third approach. While this approach achieves the advantages of the codebook-based approach, it suffers from two shortcomings. The codebook may need to be updated if the channel longterm characteristics change and the update must be reciprocal 
in the transmitter and receiver. The second is the lack of a systematic algebraic structure of the codebook. The codeword elements are random-like, which may be an issue when they are quantized for practical implementation and fed back from the receiver to the transmitter, although at a low rate.

In consideration of the foregoing, this research focuses mainly on the second approach (i.e., channel-independent and codebook-based), primarily motivated by its advantages. This approach is extensively utilized in MIMO spatial multiplexing [26] and it is also employed in LTE-A system [29]. Nevertheless, this approach did not get enough attention in the SM and SSK literature. Thus, this work introduces several codebook-based precoding methods for SSK assuming phase-only precoding. There are several reasons to prefer the phase-only precoding, in contrast to the joint amplitude and phase precoding. First, it is shown in [16] that amplitude precoding may require a large dynamic range in the precoding gain, which requires high cost linear power amplifier at the transmitter. This was also noted as the reason for phase-only precoding in [15]. Second, we find in the numerical results that four phases are sufficient for performance improvement. Increasing number of phases does not provide worthwhile gain. This considerably reduces the complexity of codebook search in the receiver and codeword scaling in the transmitter.

The contribution of this paper is dedicated for SSKMIMO system, and it can be summarized as follows:

(1) New and more accurate closed-form BER formula is derived for SSK without precoding and compared to the existing formulas in the literature.

(2) New codebooks for SSK are introduced and their performance is investigated, including the FullCombination (FC), Walsh-Hadamard (WH), QuasiOrthogonal Sequences (QOS), and Orthogonal Array Testing (OAT) codebooks. They are based on phase rotation only and require limited feedback.

(3) Iterative Search (IS) method is developed in order to solve the challenging task of selecting the best codeword iteratively from the FC codebook when its size is large.

(4) It is shown that codebooks with elements $\in\{ \pm 1, \pm j\}$ are sufficient to achieve all the performance gain from this type of codebooks. This greatly simplifies the practical implementation since channel scaling becomes a sign inversion and/or swapping real and imaginary parts.

(5) The performance of the proposed codebooks is investigated under imperfect CSI estimation and correlated channel for both Rayleigh and Nakagami fading channels.

(6) The performance of the proposed codebooks is compared to selected published performance in the literature.

The remainder of this paper is organized as follows: in Section 2, the system model of SSK-MIMO is introduced. Development of the new closed-form BER for conventional
SSK-MIMO system is presented in Section 3. Section 4 explains the codebook-based TPC and investigates the FC, WH, QOS, and OAT codebooks. Also, this section presents the IS method to select the codeword from the FC codebook. Simulation results and performance comparisons are presented in Section 5. Finally, Section 6 concludes the paper.

Notation. $(\cdot)^{*},(\cdot)^{T}$, and $(\cdot)^{H}$ indicate conjugate, transpose, and Hermitian transpose, respectively. Boldface uppercase and lowercase letters denote matrices and vectors, respectively. Italic upper or lower case letters represent the scalar variables. The notation $\operatorname{diag}(\mathbf{x})$ denotes a diagonal matrix with vector $\mathbf{x}$ in its diagonal elements. The notation $\|\cdot\|$ refers to the Euclidean norm. The gamma function of an integer is defined as $\Gamma(n+1)=n$ !, where $n$ ! is the factorial of the integer $n$.

\section{SSK-MIMO System Model}

Consider SSK-MIMO system with $N_{t}$ transmit antennas and $N_{r}$ receive antennas. Information bits are divided into sections of length $\log _{2}\left(N_{t}\right)$ bits, where we assume that $N_{t}$ is a power of 2. Each section is converted from binary to decimal to yield the index $k$ of the antenna to be activated, $k=0,1, \ldots, N_{t}-1$. The signal of SSK-MIMO systems can be expressed as follows:

$$
\mathbf{r}=\mathbf{H P x}_{k}+\mathbf{w},
$$

where $\mathbf{r}$ is the complex received signal vector and $\mathbf{H}=$ $\left[\mathbf{h}_{0}, \mathbf{h}_{1}, \ldots, \mathbf{h}_{N_{t}-1}\right]$ is the $\left(N_{r} \times N_{t}\right)$ complex channel matrix, which is known to the receiver only. $\mathbf{H}$ consists of $N_{t}$ channel vectors $\mathbf{h}_{m}$. The elements of $\mathbf{h}_{m}$ are denoted as $h_{n, m}$ which indicate the channel path gain between the $m$ th transmit antenna and the $n$th receive antenna. The elements $h_{n, m}$ are assumed to be identical and independently distributed (i.i.d.) complex Gaussian random variables with zero mean and unit variance. The $\left(N_{t} \times N_{t}\right)$ Phase-Rotation Precoding (PRP) matrix $\mathbf{P}$ has a diagonal structure to satisfy the SSK requirements of a single active antenna at a transmission time. The $\left(N_{t} \times 1\right)$ transmission vector $\mathbf{x}_{k}$ is given by

$$
\mathbf{x}_{k}=[0, \ldots, \underset{k \mathrm{th}}{1}, \ldots, 0]^{T},
$$

which indicates that only the $k$ th antenna is activated. The $\left(N_{r} \times 1\right)$ noise vector $\mathbf{w}$ is the white noise whose elements are i.i.d. complex Gaussian random variables with zero mean and variance $1 / \gamma$, where $\gamma$ is the SNR per receive antenna.

Let us define the $\left(N_{c} \times N_{t}\right)$ codebook matrix $\mathrm{C}$ that includes $N_{c}$ codewords as follows:

$$
\mathbf{C}=\left[\mathbf{p}_{0}, \mathbf{p}_{1}, \ldots, \mathbf{p}_{q}, \ldots, \mathbf{p}_{N_{c}-1}\right]^{T} .
$$

Each row in $\mathbf{C}$ represents a codeword of length $N_{t}$ that is used to scale the $N_{t}$ TAs. If the selected codeword is $\mathbf{p}_{q}$, then the precoding matrix $\mathbf{P}=\operatorname{diag}\left(\mathbf{p}_{q}\right)$. Without precoding all elements in $\mathbf{p}_{q}$ are ones. Figure 1 shows the SSK system with codebook-based TPC.

Given the estimated CSI and the utilized codeword, the receiver invokes ML detection [8] on the received vector $\mathbf{r}$ to 


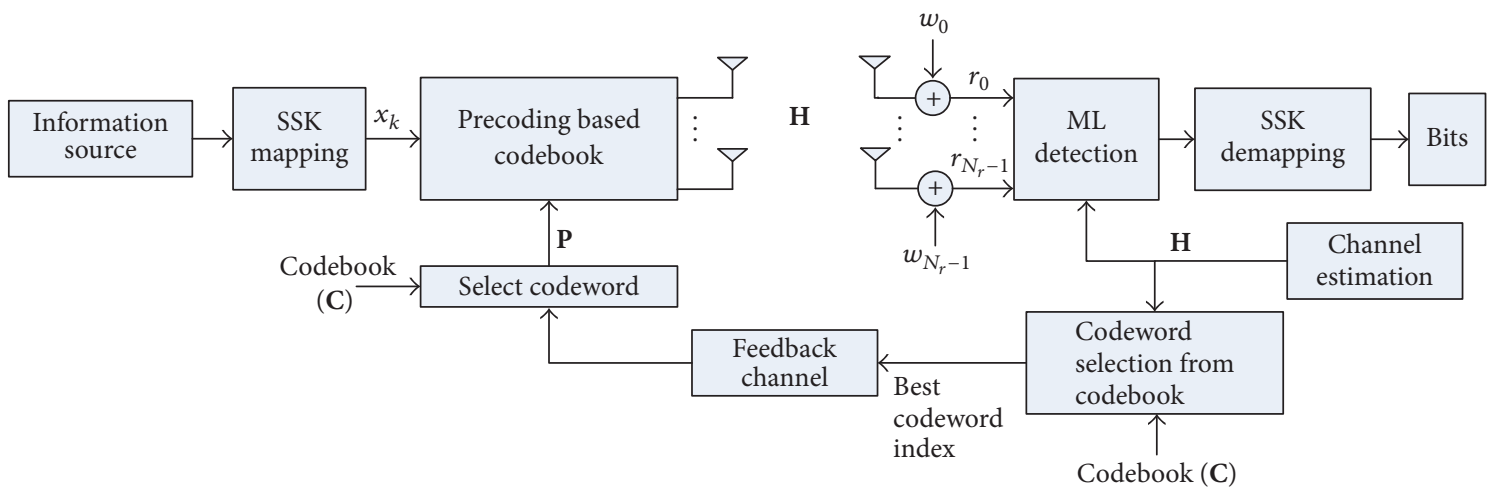

FIGURE 1: SSK-MIMO system model for codebook-based precoding.

decide the TA index. This index is demapped using decimal to binary conversion into the corresponding transmitted bits. In addition, at a reasonable rate that corresponds to the coherence time of the channel, a new codeword is selected from the codebook and its index is fed back to the transmitter. In our simulations, independent channels $\mathbf{H}$ are generated each transmission, and the best codeword is selected and used in the transmitter and receiver.

\section{Bit Error Rate for Conventional SSK-MIMO System}

The availability of a closed-form BER for the conventional SSK is very important since it provides easy and fast way for evaluating its performance with different set of parameters, comparing it with other alternative technologies and evaluating the gain realized by any enhancements that may be added to it. An upper bound for the BER of SM is widely utilized. See, for example, $[30,31]$. This upper bound can be used for SSK as well. The upper bound BER of SM in equation (18) of [30] can be specialized for SSK as follows:

$$
\begin{aligned}
P_{b} \text { (error) } \leq & \frac{N_{t}}{2}\left[\frac{1}{2}\left(1-\sqrt{\frac{\gamma}{2+\gamma}}\right)\right]^{N_{r}} \\
& \cdot \sum_{k=0}^{N_{r}-1}\left(\begin{array}{c}
N_{r}-1+k \\
k
\end{array}\right) \\
& \cdot\left[\frac{1}{2}\left(1+\sqrt{\frac{\gamma}{2+\gamma}}\right)\right]^{k} .
\end{aligned}
$$

This same formula is also available as the SSK special case of equation (17) and Table I in [31]. The upper bound of (4), which is widely used in the literature, is based on the union bound and the pairwise error probability (PEP). This bound is known to be loose at low SNR and/or small number of receive antennas [31]. To the best of the authors' knowledge, no other BER formula for SSK is available in the literature. In this section, we derive an approximation of the BER for the conventional SSK system in Rayleigh fading channel that is much more accurate than (4), specially for low SNR and small $N_{r}$.
Assume that the TA number $m$ is active. The received SSK signal is given by

$$
\mathbf{r}=\mathbf{h}_{m}+\mathbf{w}
$$

where $\mathbf{r}=\left[r_{0}, r_{1}, \ldots, r_{N_{r}-1}\right]^{T}$ and $\mathbf{h}_{m}=\left[h_{0, m}, h_{1, m}, \ldots, h_{N_{r}-1, m}\right]^{T}$ is the $m$ th channel vector in $\mathbf{H}$. Using the ML criteria, the receiver calculates the squared distances $V_{n}, n=0,1, \ldots, N_{t}-$ 1 , between the received vector $\mathbf{r}$ and all the $N_{t}$ columns of $\mathbf{H}$.

The squared distance $V_{n}$ is given by $V_{n}=\left\|\mathbf{h}_{m}-\mathbf{h}_{n}+\mathbf{w}\right\|^{2}$. The receiver selects the smallest $V_{n}$ to correspond to the active antenna in the transmitter. If the decision is correct $(n=$ $m)$, we get $Y=V_{m}=\|\mathbf{w}\|^{2}$. Symbol error occurs if $Y$ is larger than any other distance. Define $Z=\min \left(V_{n}, n=\right.$ $\left.0,1, \ldots, N_{t}-1, n \neq m\right)$. Symbol error occurs if $Z<$ $Y$. The two random variables $Y$ and $V_{n}, n \neq m$, follow $\chi^{2}$ distribution with $2 N_{r}$ degrees of freedom and statistical means $N_{r} / \gamma$ and $N_{r}(2+1 / \gamma)$, respectively [32]. Define $f_{S}(s)$ and $F_{S}(s)$, respectively, as the probability density function (pdf) and the cumulative distribution function (cdf) of a random variable $S$. To proceed further, we need the cdf of $Z$. This is very challenging since the $\chi^{2}$ random variables $V_{n}$ defined above are correlated. In [33], the pdf and cdf are derived for the maximum of two correlated Rayleigh and $\chi^{2}$ random variables. Similar derivation could be done for the minimum. However, in SSK, we are interested in large number of random variables, corresponding to large number of TAs. Finding the pdf or cdf of the minimum of more than two correlated $\chi^{2}$ random variables is a formidable task. To simplify analysis, we invoke the approximation that the random variables $V_{n}$ are independent, $n=0,1, \ldots, N_{t}-1$. Fortunately, we will find that this approximation still yields accurate BER results. by

Using this approximation, the symbol error rate is given

$$
\begin{aligned}
P_{\text {sym }} \text { (error) } & =\operatorname{Pr}(Z<Y) \\
& =\int_{y=0}^{\infty} \int_{z=0}^{y} f_{Y}(y) f_{z}(Z) d z d y \\
& =\int_{0}^{\infty} f_{Y}(y) F_{Z}(y) d y .
\end{aligned}
$$


The cdf of $Z$ is related to the cdf of $V_{n}$ as $F_{Z}(y)=1-(1-$ $\left.F_{V}(y)\right)^{N_{t}-1}$. The pdf of $Y$ and the cdf of $V_{n}$ can be written, respectively, as follows [32]:

$$
\begin{aligned}
& f_{Y}(y)=\frac{y^{N_{r}-1} \gamma^{N_{r}} \exp (-y \gamma)}{\Gamma\left(N_{r}\right)}, \quad y>0, \\
& F_{V}(y)=1-\exp \left(-\frac{y \gamma}{1+2 \gamma}\right) \sum_{k=0}^{N_{r}-1} \frac{1}{k !}\left(\frac{y \gamma}{1+2 \gamma}\right)^{k}, \quad y>0 .
\end{aligned}
$$

Using (7) in (6) and processing the integration, we find the symbol error rate as

$$
\begin{aligned}
& P_{\text {sym }}(\text { error })=1-\frac{(1+2 \gamma)^{N_{r}}}{\Gamma\left(N_{r}\right)} \\
& \quad \cdot \int_{0}^{\infty} y^{N_{r}-1} \exp \left(-\left(2 \gamma+N_{t}\right) y\right)\left(\sum_{k=0}^{N_{r}-1} \frac{y^{k}}{k !}\right)^{N_{t}-1} d y .
\end{aligned}
$$

To express (8) in a closed form, we use the following expansion:

$$
\left(\sum_{k=0}^{N_{r}-1} \frac{y^{k}}{k !}\right)^{N_{t}-1}=\sum_{k=0}^{L} \beta_{k} y^{k}
$$

where $L=\left(N_{t}-1\right)\left(N_{r}-1\right)$ and $\beta_{k}$ is the set of coefficients in the expansion. They can be found as in [34], section 1.9.60. Using this expansion, we get the closed-form BER for the symbols error rate as follows:

$$
\begin{aligned}
& P_{\text {sym }} \text { (error) } \\
& \qquad=1-\frac{1}{\Gamma\left(N_{r}\right)}\left(\frac{1+2 \gamma}{N_{t}+2 \gamma}\right)^{N_{r}} \sum_{k=0}^{L} \beta_{k} \frac{\Gamma\left(k+N_{r}\right)}{\left(N_{t}+2 \gamma\right)^{k}} .
\end{aligned}
$$

Now, since there is no Gray coding, symbol error leads to bit errors in any of the other $N_{t}-1$ symbols with equal probability. An SSK symbol includes $B=\log _{2}\left(N_{t}\right)$ bits. When a symbol error occurs the number of symbols with $m$ bit error is $\left(\begin{array}{c}B \\ m\end{array}\right)$. Hence, given a symbol error, the average number of bit errors is $\left(1 /\left(N_{t}-1\right)\right) \sum_{m=1}^{B} m\left(\begin{array}{l}B \\ k\end{array}\right)=B N_{t} / 2\left(N_{t}-1\right)$. Consequently, the BER is related to the SER of (10) by

$$
P_{\text {bit }} \text { (error) }=P_{\text {sym }}(\text { error }) \frac{N_{t}}{2\left(N_{t}-1\right)} \text {. }
$$

A special case of interest is when $N_{r}=1$. In this case (4) simplifies to

$$
P_{b}(\text { error }) \leq \frac{N_{t}}{4}\left(1-\sqrt{\frac{\gamma}{2+\gamma}}\right),
$$

while the closed-form (11) reduces to

$$
P_{b} \text { (error) }=\frac{N_{t}}{2 N_{t}+4 \gamma} \text {. }
$$

Clearly, the upper bound (12) is very loose, as it linearly increases with $N_{t}$ and could go higher than 1 . In contrast, the BER of (13) increases slowly with $N_{t}$ and is upper bounded by 0.5 when $N_{t}$ is large. It is shown in the numerical results that the BER of (11) and (13) provides a very close agreement with simulation.

\section{Precoding Aided SSK-MIMO Systems}

In the previous section, the BER is derived for the conventional SSK system, that is, without precoding. The CSI is assumed unknown to the transmitter. However, if the transmitter has knowledge about the downlink CSI, it can preprocess the transmitted signal to reduce the BER. Conditioned on the channel gains $h_{i j}$, the BER of SSK is upper bounded by the following [3]:

$$
\begin{aligned}
& P_{b}(\text { error }) \leq \frac{2}{N_{t} \log _{2} N_{t}} \\
& \quad \cdot \sum_{i=0}^{N_{t}-1} \sum_{j=i+1}^{N_{t}-1} D\left(\mathbf{h}_{i}, \mathbf{h}_{j}\right) Q\left(\sqrt{\frac{\gamma\left\|\mathbf{h}_{i}-\mathbf{h}_{j}\right\|^{2}}{2}}\right),
\end{aligned}
$$

where $D\left(\mathbf{h}_{i}, \mathbf{h}_{j}\right)$ is the number of bit errors when the channel vector $\mathbf{h}_{i}$ (the $i$ th column of $\mathbf{H}$ ) is wrongly detected as $\mathbf{h}_{j}$, and $Q(\cdot)$ is the $Q$-function. The upper bound (14) consists of a weighted sum of $Q$-functions whose arguments are scaled squared Euclidean Distances (EDs) between pairs of channel vectors. Since $Q(x)$ rapidly decays with increasing $x$, the upper bound (14) is dominated by the smallest MED $\left\|\mathbf{h}_{i}-\mathbf{h}_{j}\right\|^{2}$ (for simplicity, for the remainder of the paper, we refer to the squared value of the MED as the MED). By precoding (i.e., scaling) the $i$ th transmit antenna by the $i$ th element of a codeword $\mathbf{p}_{k}=\left[p_{k, 0}, p_{k, 1}, \ldots, p_{k, N_{t}-1}\right]^{T}$, the precoded MED $\left\|p_{k, i} \mathbf{h}_{i}-p_{k, j} \mathbf{h}_{j}\right\|^{2}, i \neq j$, can be increased, leading to a reduced BER in (14). Therefore, it is a common practice to find the best codeword $\mathbf{p}_{b}$ that maximizes the MED. In this section, we focus on constructing an $\left(N_{c} \times N_{t}\right)$ codebook matrix $\mathbf{C}$ consisting of $N_{c}$ codewords, where $\mathbf{C}$ is known to the transmitter and receiver. From $\mathbf{C}$, the best codeword $\mathbf{p}_{b}$ is selected by testing all $N_{c}$ codewords and selecting the one that maximizes the MED as follows:

$$
\mathbf{p}_{b}=\arg \max _{\mathbf{p}_{k} \in \mathbf{C}}\left(\min _{i \neq j}\left(\left\|p_{k, i} \mathbf{h}_{i}-p_{k, j} \mathbf{h}_{j}\right\|^{2}\right)\right) .
$$

The receiver feeds back the index $b$ of this codeword to the transmitter using $N_{\mathrm{fb}}$ bits. The following subsections present several codebooks for SSK. All codebooks assume phase-only precoding where the codeword elements are selected from the set $\{\exp (j 2 \pi m / M), m=0,1, \ldots, M-1\}$, where $M \geq 1$ is the number of possible phases.

4.1. Full-Combinations (FC) Codebook. In this case, the gains $p_{k, i}$ take all possible combinations of the $M$ phases with $p_{k, i}=\exp \left(j 2 \pi m_{k, i} / M\right)$. The first antenna is a reference with $m_{k, 0}=0$ and $p_{k, 0}=1$ for all $k$. The remaining vector $\left[m_{k, 1}, m_{k, 2}, \ldots, m_{k, N_{t}-1}\right]^{T}$ is the conversion of the decimal codeword index $k$ into a Base- $M$ number with $N_{t}-1$ digits. Hence, the codebook size is $N_{c}=M^{\left(N_{t}-1\right)}$ codewords, each with length $N_{t}$ gains. With $p_{k, 0}=1$, each codeword is unique; that is, no codeword is a phase rotation of another codeword. The required number of feedback bits is $N_{\mathrm{fb}}=\left\lceil\left(N_{t}-1\right) \log _{2} M\right\rceil$, where each transmit antenna, 
except the first antenna, needs $\log _{2} M$ bits to select the gain $p_{k, i}$. This codebook is used as a reference for the other proposed methods in this paper since it provides the best BER performance. However, sequential search for the best codeword using (15) is practical only for small $N_{t}$ and $M$. For example, for $N_{t}=16$ and $M=4$, the number of codewords is $N_{c}=4^{15}$, which is formidable to search. It is important to note that although codewords are unique, several codewords yield the same MED. Hence, full search of the codebook is not necessary. We show below an iterative method to find the best codeword in the FC codebook and achieve the same BER performance of sequential search.

4.2. Walsh-Hadamard (WH) Codebook. When $N_{t}$ is large we can still search a large FC codebook efficiently using the iterative method described below. However, the number of feedback bits using the FC codebook may be unacceptable in some applications. In this case, a short codebook is desirable. The codebook must provide sufficient codeword diversity with large dissimilarity. This motivates us to investigate orthogonal codebooks. An interesting short codebook to investigate is the well-known binary Walsh-Hadamard (WH) square matrix [35] with size $N_{t}$. The number of codewords is $N_{c}=N_{t}$, requiring only $N_{\mathrm{fb}}=\log _{2} N_{t}$ feedback bits. The $\mathrm{WH}$ codebook is relatively short and the sequential search is simple ( $p_{i}$ and $p_{j}$ are only sign changes in (15)). The disadvantage is the limitation of two-phase codewords with \pm 1 values only. However, it is shown in the numerical results that the WH codebook provides considerable BER reduction compared to the case without precoding, which makes it an attractive solution.

4.3. Quasi-Orthogonal Sequences (QOS) Codebook. To circumvent the two-phase limitation of the WH codebook, we can use Quasi-Orthogonal Sequences (QOS) codebook. QOSs are used in Code Division Multiple Access (CDMA) systems to augment the WH sequences and accommodate more users $[36,37]$. There are families of real and complex QOSs. Here, we are interested in a codebook whose elements are 4-phase $\in\{ \pm 1, \pm j\}$. QOS codebook is generated using the WH codebook and a set of $N$ masking sequences with size $N_{t}$. Each masking sequence is element-by-element multiplied by each WH codeword to generate a QOS codebook with size $N \times N_{t}$. References [36, 37] describe methods for generating such masking sequences. Masking sequences with size $2^{m}, m=3$ to 8 , are listed in [36], Table VI, which we employ in this paper. Using these masking sequences, we can generate QOS codebooks of the desired size. In this paper we use QOS codebook with size $N_{c}=4 \times N_{t}$ (i.e., using 4 masking sequences), which we found to provide close performance to the FC codebook. Obviously, the codebook size can be reduced. The choice of $N_{c}$ is a tradeoff between BER performance and number of feedback bits.

4.4. Orthogonal Array Testing (OAT) Codebook. Orthogonal Array Testing (OAT) matrix is a statistical method mainly used for experimental testing in industrial engineering to avoid the difficulty of testing all possible combinations of
TABLE 1: Codebook size and feedback bits for proposed codebooks.

\begin{tabular}{lcc}
\hline Codebook & Size, $N_{c}$ & Feedback bits \\
\hline FC & $M^{\left(N_{t}-1\right)}$ & $\left\lceil\left(N_{t}-1\right) \log _{2} M\right\rceil$ \\
WH & $N_{t}$ & $\log _{2} N_{t}$ \\
QOS & $4 \times N_{t}$ & $2+\log _{2} N_{t}$ \\
OAT & $M N_{t}$ & $\log _{2} N_{t}+\left\lceil\log _{2} M\right\rceil$ \\
\hline
\end{tabular}

experimental factors when each factor has various possible levels [38]. The methodology of OAT is based on the selection of a concise and effective set from the full-combinations such that this set is enough to consider all the effects of each factor [38]. Also, this selection guarantees testing all the pairwise combinations of all cases in the set. Typically, OAT is described with the notation $L_{\text {Runs }}$ (Levels ${ }^{\text {Factors }}$ ), where Runs, Levels, and Factors are integers. The integer Runs is the total number of chosen combinations to test (number of rows in the matrix). The integer Factors is the number of factors to test in each combination (number of columns in the matrix) [39]. Hence, the matrix size is (Runs $\times$ Factors) with entries from 0 to Levels -1 , where the Levels is the number of levels to test for each factor. For efficient testing, the integer Runs typically satisfies Runs $\ll$ Levels $^{\text {Factors }}$. A detailed description of the properties and implementation of OAT is available in [38, 40-42].

While OAT is invented for experimental testing of an industrial design with sufficient test coverage, it found applications in communication systems [39]. In this paper, the concept of OAT is utilized to design the precoding codebook where the integers Factors, Levels, and Runs represent the number of TAs $\left(N_{t}\right)$, the number of phase rotations $(M)$, and the codebook size $\left(N_{c}\right)$, respectively. Therefore, the OAT codebook size is $\left(N_{c} \times N_{t}\right)$. The codebook entries are integers $m_{k, i}$ which take values from 0 to $M-1$. Similar to the FC codebook, these entries are converted into the $M$ phasors $p_{k, i}$. The size of OAT codebooks implemented in this paper is the typical size $N_{c}=M N_{t}$. Hence, the number of feedback bits is $N_{\mathrm{fb}}=\log _{2} N_{t}+\log _{2} M$, which is much smaller than the FC codebook for large $N_{t}$. The construction of the OAT codebooks for $M=2$ with $N_{t}=8$ and $N_{t}=16$ is based on the tables available in [43]. The codebooks for $M=4$ with $N_{t}=8$ and $N_{t}=16$ are based on the tables available in [44] and [45], respectively. Other codebook sizes are also available in corresponding literature. The design and structure of the OAT codewords allow us to sufficiently test each channel realization $\mathbf{H}$ using (15) to find a codeword that maximizes the MED. Indeed, it is shown in the numerical results that the OAT codebook provides BER performance that is close to the FC codebook.

Table 1 compares the number of codewords to search and the number of feedback bits for the methods described above.

4.5. Iterative Search (IS) Algorithm. Iterative algorithms have been used previously in the literature to find precoders for SM or SSK. For example, in [13], the max-min algorithm is approximated by a convex function that is iteratively solved using an optimization tool. In [15], a precoder formulation 
for the simple case of $N_{t}=2$ is first found. This formulation is iteratively used for the case of $N_{t}>2$. In [17], a similar approach is used where a solution for $N_{t}=2$ is extended to $N_{t}>2$ in an iterative manner. These algorithms are not codebook-based since they belong to the first approach described in the introduction of this paper. For this reason, in this paper, we present a codebook-based iterative algorithm to select the best codeword from the FC codebook. To the best of the authors' knowledge no such algorithm is available in the literature.

As mentioned above, the FC codebook provides the best performance since it covers all possible phase-rotation combinations. However, for large $N_{t}$, its size is too large and searching all codewords may be formidable. Here, we present an efficient method to find the best codeword from the FC codebook using an iterative procedure, instead of sequentially searching all codewords. In short, given the channel matrix $\mathbf{H}=\left[\mathbf{h}_{0}, \mathbf{h}_{1}, \ldots, \mathbf{h}_{N_{t}-1}\right]$, the process is as follows: in each iteration, we find the MED and the corresponding pair of channel vectors. Then, one of the two channel vectors from the pair, say $\mathbf{h}_{k}$, is phase rotated. In the following iteration, the new MED and the corresponding pair of channel vectors are found. The new pair may or may not be the same pair of the previous iteration. If the new MED is larger, the accumulated phase rotation performed on $\mathbf{h}_{k}$ is stored as $\alpha_{k}$. The iterations are repeated till the MED stops increasing or the maximum number of iterations is reached.

Specifically, the algorithm is described in detail as follows.

Initialization Phase. $\left\{\theta_{l}=0, \quad l=0,1, \ldots, N_{t}-1\right\}, \phi=2 \pi / M$, and $D=0$.

Iterations Phase. Given $\mathbf{H}$, in the $n$th iteration, the following is performed:

(1) Find the EDs between all $N_{t}\left(N_{t}-1\right) / 2$ pairs of channel vectors.

(2) Find the MED, say $d$, and the corresponding pair of channel vectors, say $\mathbf{h}_{i}$ and $\mathbf{h}_{j}, i \neq j$.

(3) If $d$ is larger than $D$, update the MED $D=d$, and the accumulated phase rotation $\left\{\alpha_{l}=\theta_{l}, l=\right.$ $\left.0,1, \ldots, N_{t}-1\right\}$. Otherwise, no update is done.

(4) Select one of the two channel vectors $\mathbf{h}_{i}$ or $\mathbf{h}_{j}$ corresponding to the MED in Step (2) to be phase rotated by the angle $\phi$ (i.e., select $k=i$ or $k=j$ ). Selection is as follows:

(i) Select $k \neq 0$, since antenna 0 is reference and not precoded.

(ii) If $i \neq 0$ and $j \neq 0$, select $k$ that was not selected in the previous iteration.

(iii) Else, select $k=i$ or $j$ randomly with equal probability.

(5) Phase rotate the $k$ th channel vector by angle $\phi$; that is, set $\mathbf{h}_{k}=e^{j \phi} \mathbf{h}_{k}$. Also update the corresponding accumulated phase rotation $\theta_{k}=\left(\theta_{k}+\phi\right)_{\bmod 2 \pi}$.

(6) Go to Step (1).
The iterative search continues till the MED does not change for $K$ successive iterations or the maximum number of iterations is reached. The output of the algorithm is the set of phase rotations per antenna $\left\{\alpha_{l}, l=0,1, \ldots, N_{t}-1\right\}$, where $\alpha_{0}=0$ and the remaining phases are from the set $\{m \phi, m=0,1, \ldots, M-1\}$. The selected codeword is $\left\{e^{j \alpha_{l}}, l=\right.$ $\left.0,1, \ldots, N_{t}-1\right\}$. Figure 2 shows a flowchart of the algorithm.

We found that a maximum number of iterations of $10 N_{t} \log _{2} M$ is sufficient to reach the best codeword with very high probability. We also take $K=20$. Note that the IS algorithm considerably reduces the number of MED calculations. For example, if $N_{t}=8$ and $M=4$, searching the FC codebook requires 16384 MED calculations, while the IS requires at most 160 calculations.

\section{Numerical Results and Discussions}

In this section, we present the BER of the new closed form (11) as well as the performance of the proposed codebookbased PRP methods in uncorrelated Rayleigh fading channel. Moreover, to verify the robustness of the proposed methods, we show results for correlated fading, CSI estimation error, and Nakagami- $m$ fading channels with $m>1$ (i.e., less severe fading than Rayleigh). In all figures, the conventional SSK without precoding is denoted as just SSK. Unless otherwise mentioned, the system is simulated for uncorrelated Rayleigh fading channel. Finding a closed form expression for the BER with codebook-based precoding is very difficult since the statistics of the effective channel, $\mathbf{H P}$ in (1), is prohibitive. Hence, in this case, we rely on computer simulation. When including results from the literature, we plot the BER versus the SNR per receive antenna, $\gamma$. Otherwise, we plot versus the $E_{b} / N_{o}$ per receive antenna, which simplifies comparison with traditional digital modulation techniques in any textbook and provides fair comparison between systems with different $N_{t}$. They are related as $\gamma=E_{b} / N_{o}+10 \log _{10}\left(\log _{2}\left(N_{t}\right)\right) \mathrm{dB}$. This is important to consider when comparing different figures.

Figure 3 shows the BER performance versus SNR for SSK without precoding (i.e., no phase changes and $M=1$ ) where $N_{t}=16$. The figure compares the BER of the proposed closed form (11), the closed form (4) of [30,31], and computer simulation. It is clear from the figure that when $N_{r}=1$ and $N_{r}=2$, the proposed form provides much closer results to the simulation, especially at low SNR, where the closed form of $[30,31]$ provides a loose bound. At high SNR and large $N_{r}$ we note a diminishing difference among the proposed approximation (11), the upper bound (4), and the simulation results. This shows the higher accuracy of the new BER closed form presented in this paper. It is interesting to note in Figure 3 that the approximation of (11) provides an upper bound of the BER. However, rigorous proof of this note was not possible due to the difficulty of evaluating the behavior of the pdf of the random variables $Y$ and $Z$ (in Section 3) when the involved random variables are correlated.

Figure 4 shows the system performance with FC codebook for $N_{t}=4$. The number of phases is $M=2,4$, and 8 , corresponding to codebook size $N_{c}=8,64$, and 512, respectively. From Figure 4, we can see that, for $M=2$, 


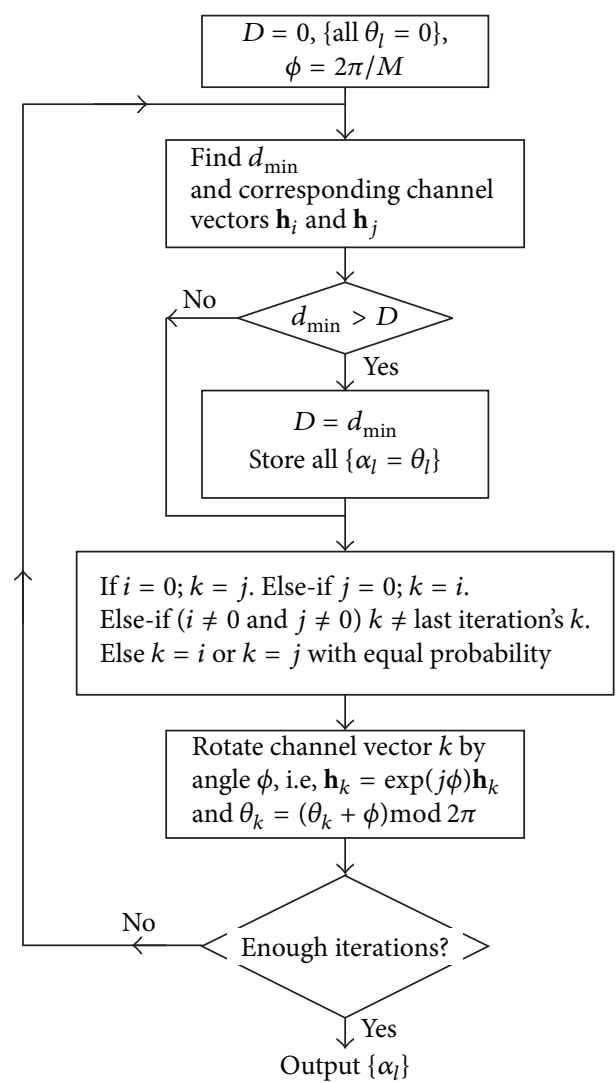

Figure 2: Iterative Search (IS) algorithm.

a gain of almost $5 \mathrm{~dB}$ at BER of $10^{-3}$ is achieved, compared to conventional SSK. The gain increases at lower BER. An additional gain of $1 \mathrm{~dB}$ is achieved by increasing the number of phases to $M=4$. However, increasing the number of phases to $M=8$ yields insignificant gain. Therefore, it is clear that a codebook with $M=4$ phases is sufficient. Also, the results of the Maximum Minimum Distance (MMD) and Guaranteed Euclidean Distance (GED) algorithms from $[12,13]$ are shown for comparison. Both methods require full knowledge of CSI at the transmitter and receiver to optimize gain and phase precoding. While they indeed outperform the FC codebook, which requires limited CSI at the transmitter, the difference is only about $0.5 \mathrm{~dB}$ at BER of $10^{-3}$ and slightly increases at lower BER. This indicates that the proposed phase rotation only precoding is very promising.

Figure 5 shows the BER versus the number of phases $M$ for systems with $N_{t}=4,8$, and 16 . The BER is plotted for $E_{b} / N_{o}=7$ and $9 \mathrm{~dB}$. Increasing the number of phases from 1 (no precoding) to 2 considerably reduces the BER. Further $\mathrm{BER}$ reduction is achieved by increasing $M$ up to 4 . However, increasing the number of phases beyond $M=4$ does not yield much BER reduction. This indicates that when phaseonly precoding is used, it is sufficient to employ 4 phases. Therefore, for the remainder of the numerical results, we consider $M=2$ and $M=4$ phases only. We also note from the figure that the BER of $N_{t}=4$ and $N_{t}=8$ with precoding

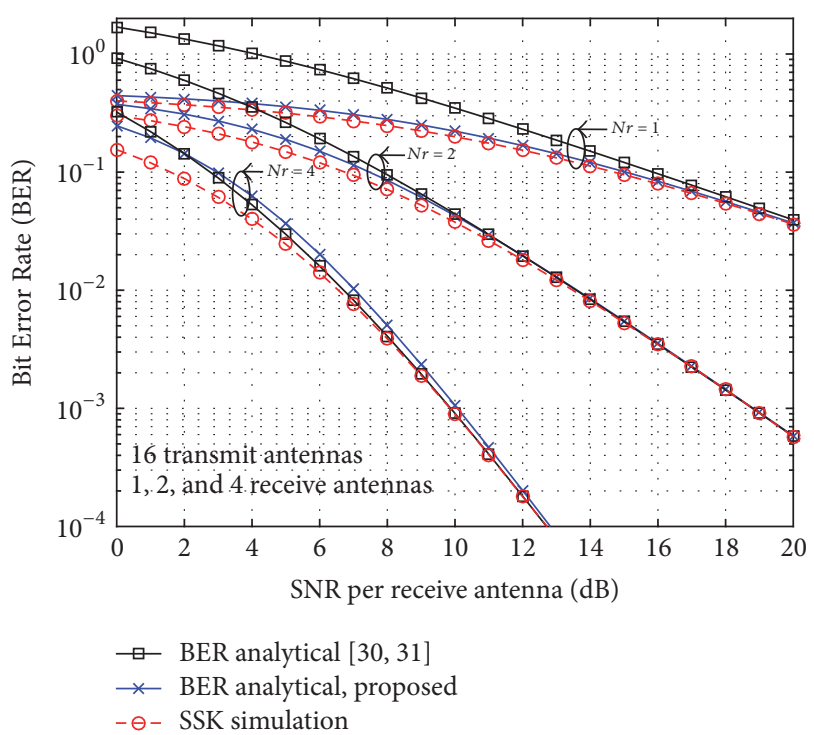

FIGURE 3: BER comparison between the proposed closed form (11), the closed form (4), and simulation results for SSK with $N_{t}=16$ and $N_{r}=1,2$, and 4 .

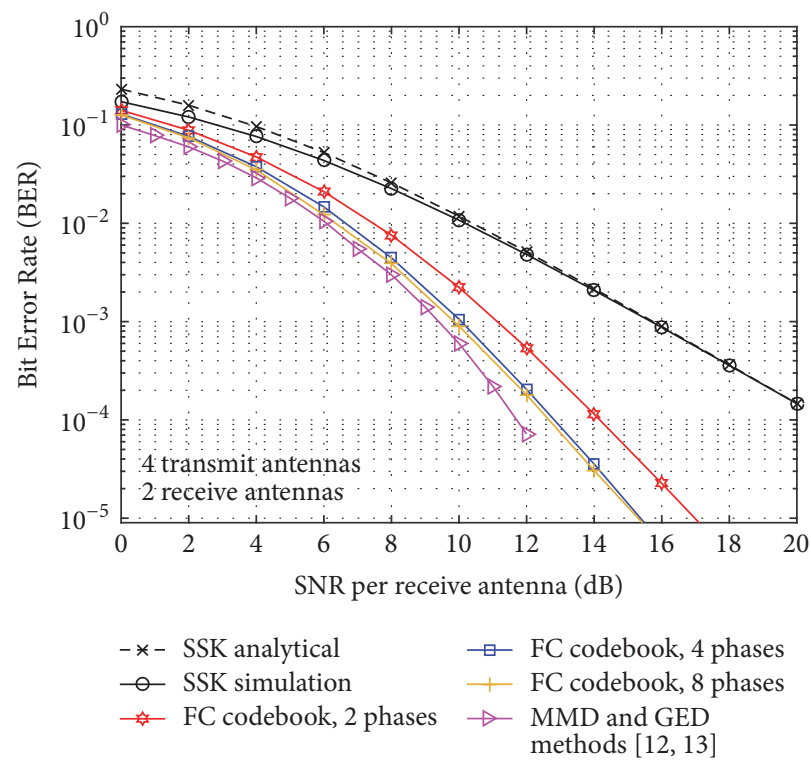

FIGURE 4: BER performance versus SNR for FC codebook with $M=$ 2,4 , and 8 phases and for $N_{t}=4$ and $N_{r}=2$.

are close for the same $E_{b} / N_{o}$, while the latter system provides higher bit rate.

Figure 6 presents a performance comparison between all the proposed codebooks in this paper for $N_{t}=8$ and $N_{r}=2$. The FC codebook serves as the lower limit of BER that can be achieved by a phase-rotation-only codebook. For all methods a significant gain can be achieved with $M=2$ phases, with further gain at $M=4$ phases. Moreover, it is clear from Figure 6 that for $M=2$ the WH and OAT codebooks provide close BER performance to the FC codebook. For $M=2$ phases, the OAT, whose codebook size $N_{c}=16$, provides very slight performance gain compared to the WH, with $N_{c}=8$. 


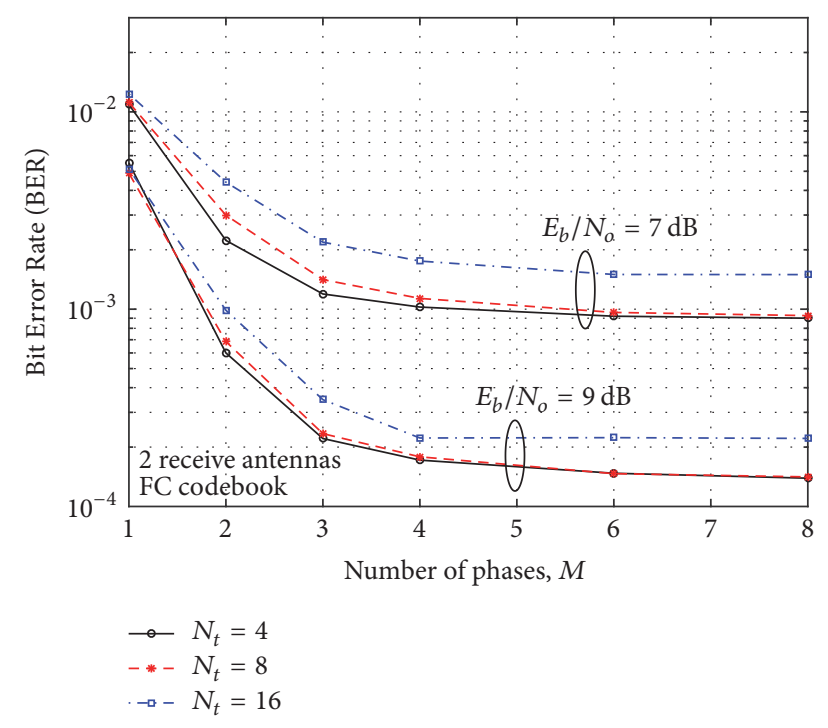

FIGURE 5: BER performance versus number of phases $M$ with FC codebook for $N_{t}=4,8$, and 16 and $N_{r}=2$.

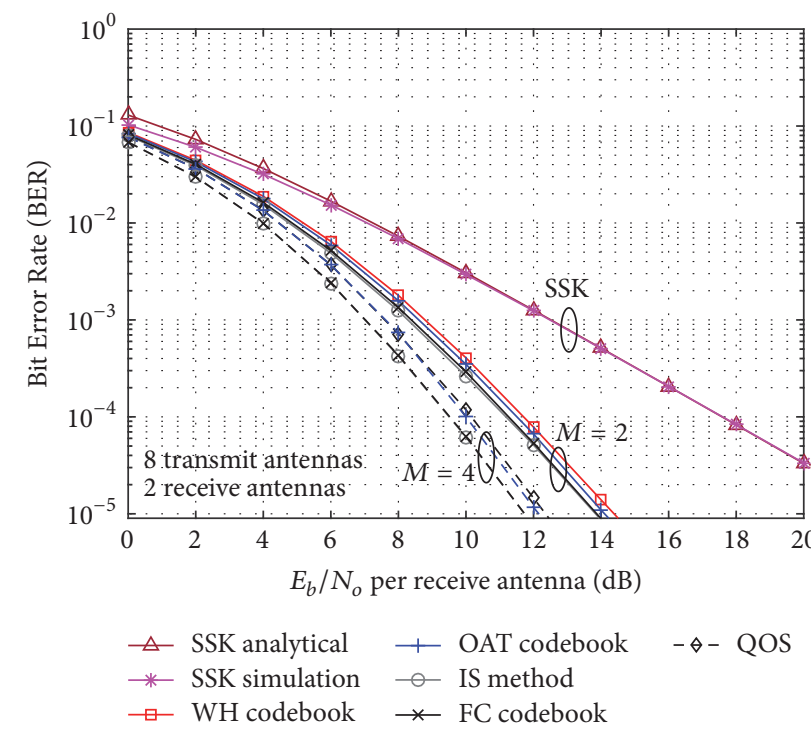

FIGURE 6: BER of all proposed codebooks with $N_{t}=8, N_{r}=2$. Solid lines indicate employing $M=2$ phases, while dashed lines indicate $M=4$ phases.

Hence, the WH codebook is preferable. For $M=4$ phases, QOS and OAT provide almost the same performance, which is again very close to the FC codebook. Both codebooks have the same size $N_{c}=32$. For both $M=2$ and $M=4$, the IS method achieves the same performance of FC codebook, which indicates that the IS algorithm can reach the best codeword from the FC codebook.

Figure 7 provides performance comparison between the proposed methods in this paper and related methods in the literature [13,22] with $N_{t}=16$; and both $N_{r}=2$ and $N_{r}=4$. For the proposed methods we consider $M=4$ phases. Since the FC codebook size $N_{c}=M^{15}$ is too large, the IS method is used. We compare the different methods at BER $=10^{-3}$.

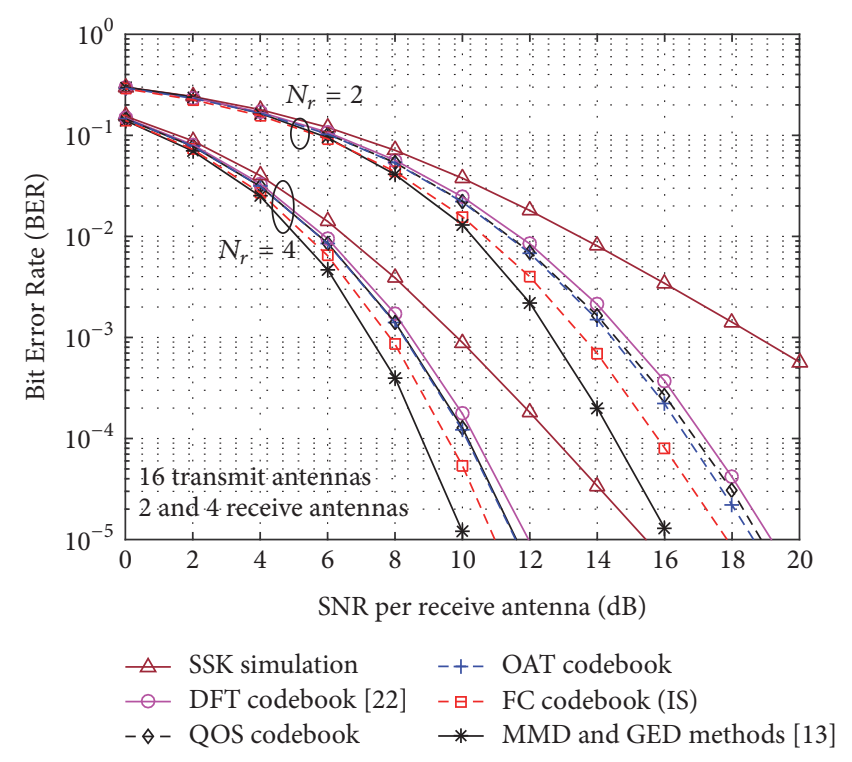

FIGURE 7: BER performance comparison with related methods in $[13,22]$ with $N_{t}=16$ and $N_{r}=2$ and $N_{r}=4$.

For $N_{r}=2$ the MMD and GED methods of [13] provide the best performance, outperforming the FC codebook by $1 \mathrm{~dB}$. The FC codebook outperforms the OAT and the QOS by another $1 \mathrm{~dB}$. The OAT and QOS provide almost the same performance. Then the DFT codebook proposed in [22] shows the least performing precoder. Obviously the conventional SSK shows the least performance.

The order of performance between different methods did not change with $N_{r}=4$. However, due to the higher diversity gain, the gap among the different methods becomes smaller. The small gap between the proposed methods and the optimized MMD and GED confirms that the proposed methods are attractive and practical when only limited knowledge of CSI at the transmitter is required (i.e., codebook index) and simpler processing is desired.

It is known that TA correlation has a detrimental effect on the performance of SM in general and SSK in particular. Therefore, it is important to investigate the performance of the proposed codebook precoding methods in this case. The channel matrix of (1) is rewritten as $\mathbf{H}=\mathbf{H}_{w} \mathbf{R}^{1 / 2}$, where $\mathbf{H}_{w}$ is the uncorrelated channel $\mathbf{H}$ in (1), while $\mathbf{R}$ is the channel correlation matrix at the transmitter. It is common in the literature $[3,13,19]$ to employ the simple correlation matrix with elements $\rho_{i, j}=\rho^{|i-j|}$ where $i$ and $j$ are indexes of TAs in a linear array and $\rho \in[0,1]$. Figure 8 illustrates the effect of the correlation coefficient on the BER at $E_{b} / N_{o}=12 \mathrm{~dB}$. It is clear that the proposed methods are still effective under correlated channel, where the BER reduction in comparison to the conventional SSK is still considerable even in the high correlation scenarios. The FC, WH, and OAT codebooks are able to break the channel correlation and allow the receiver to distinguish the transmitting antenna with higher probability than without precoding. Also, using $M=4$ phases still provides considerably better performance than $M=2$ phases. 


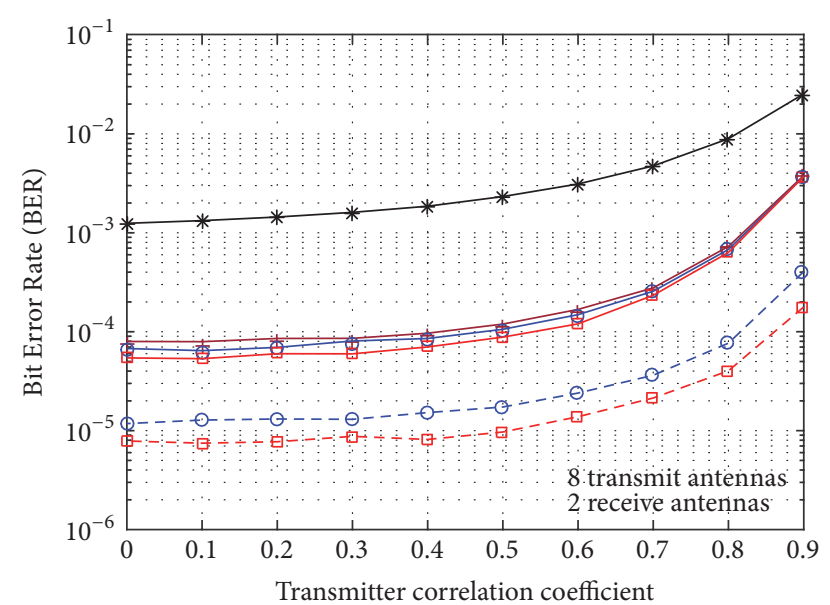

* SSK simulation - $\quad-$ OAT codebook

FIGURE 8: BER versus TA Correlation Coefficient $(\rho)$ with $N_{t}=8$ and $N_{r}=2$, in Raleigh fading channels at $E_{b} / N_{o}=12 \mathrm{~dB}$. Solid lines indicate $M=2$ and dashed lines indicate $M=4$.

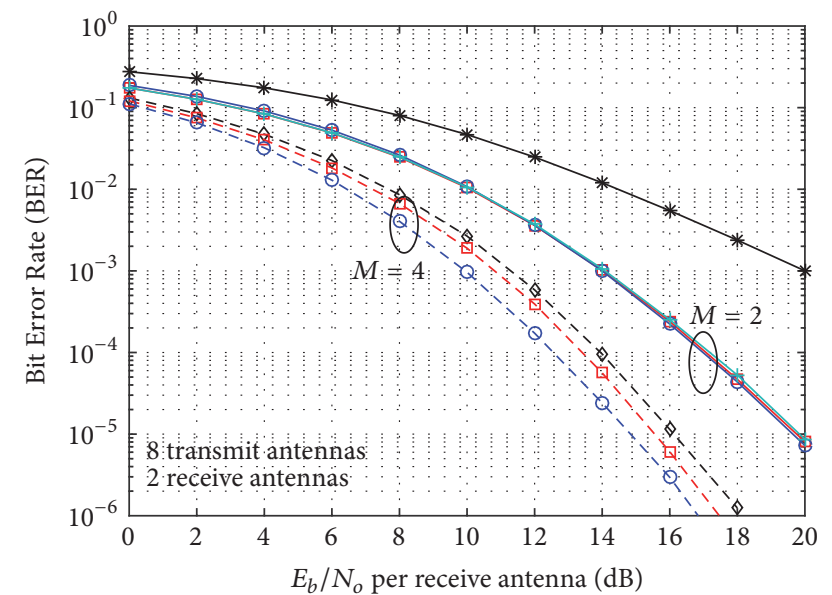

$$
\begin{array}{ll}
\text { * SSK simulation } & + \text { WH codebook } \\
- \text { - OAT codebook } & -\diamond-\text { QOS codebook } \\
- & \text { FC codebook }
\end{array}
$$

Figure 9: BER versus $E_{b} / N_{o}$ with $N_{t}=8$ and $N_{r}=2$ in correlated Raleigh channels $(\rho=0.9)$. Solid lines indicate $M=2$ phases and dashed lines indicate $M=4$ phases.

Furthermore, Figure 9 shows the BER at $\rho=0.9$. Comparing to Figure 6, it can be seen that all methods suffer from considerable performance degradation. However, codebook precoding still provides considerable gain compared to the conventional SSK. With $M=2$ all the proposed codebooks provide very similar performance, while for $M=4$ the FC codebook provides about $1 \mathrm{~dB}$ gain compared to the OAT, which provides about $0.5 \mathrm{~dB}$ gain compared to the QOS. These results confirm the applicability of the codebooks at high correlation scenarios. It is also clear that $M=4$ phases are important to reduce the channel correlation.

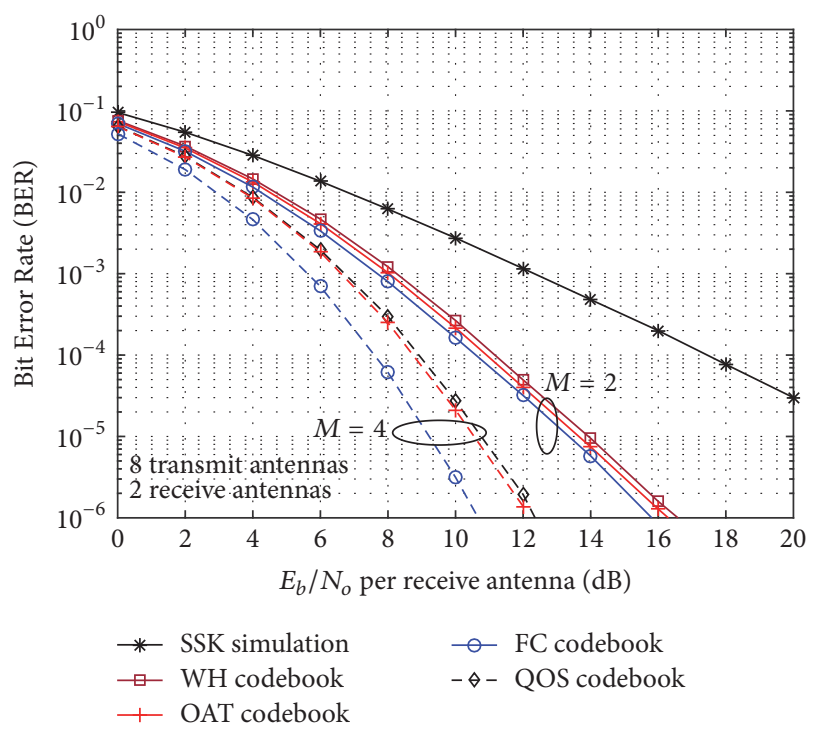

FIGURE 10: BER performance versus $E_{b} / N_{o}$ with $N_{t}=8$ and $N_{r}=2$ in uncorrelated Nakagami- $m$ fading channels $(m=2)$. Solid lines indicate $M=2$ phases and dashed lines indicate $M=4$ phases.

Figure 10 shows the BER performance of the proposed methods in Nakagami- $m$ fading channels with $m=2$, which typically causes less performance degradation than Rayleigh fading $(m=1)$. This is clear in our results when we compare Figures 6 and 10. It can also be seen that the proposed precoding methods improve the performance considerably in Nakagami fading. An interesting observation is that the gain achieved by the precoding methods, compared to conventional SSK, is higher in Nakagami fading than Rayleigh fading. In Figure 6, for example, the FC codebook, with $M=4$ phases, provides a gain of about $5.4 \mathrm{~dB}$ at BER $=10^{-3}$. The corresponding gain in Nakagami fading channel in Figure 10 is about $6.6 \mathrm{~dB}$. Similar difference can be noticed for the other proposed codebooks. This indicates the higher benefit of the proposed methods in Nakagami fading. Although not shown in the paper, the gain due to precoding increases as $m$ increases. As $m$ increases the randomness of the Nakagami channel decreases, which weakens the ability of the SSK receiver to distinguish different transmitting antennas. Hence, the precoding helps differentiate the channel gains.

Figure 11 demonstrates the effect of imperfect CSI at the receiver due to CSI estimation error. The purpose is to make sure that the proposed methods still work with CSI errors. We use the imperfect CSI model employed in $[3,17]$. The estimated channel is modeled as $\mathbf{H}_{\text {est }}=\mathbf{H}+\mathbf{H}_{\text {error }}$, where elements of $\mathbf{H}_{\text {error }}$ are i.i.d. complex Gaussian random variables with zero mean and variance $1 / \gamma$. This model considers that CSI accuracy improves as SNR increases. The erroneous channel $\mathbf{H}_{\text {error }}$ is used by the receiver to select the codeword that the transmitter will use. It is also used by the receiver for ML detection of the TA index. To avoid crowding the figure we show results for the FC and OAT codebooks with $M=2$ and $M=4$ phases. It can be seen from Figure 11 that the conventional SSK as well as the shown precoding 


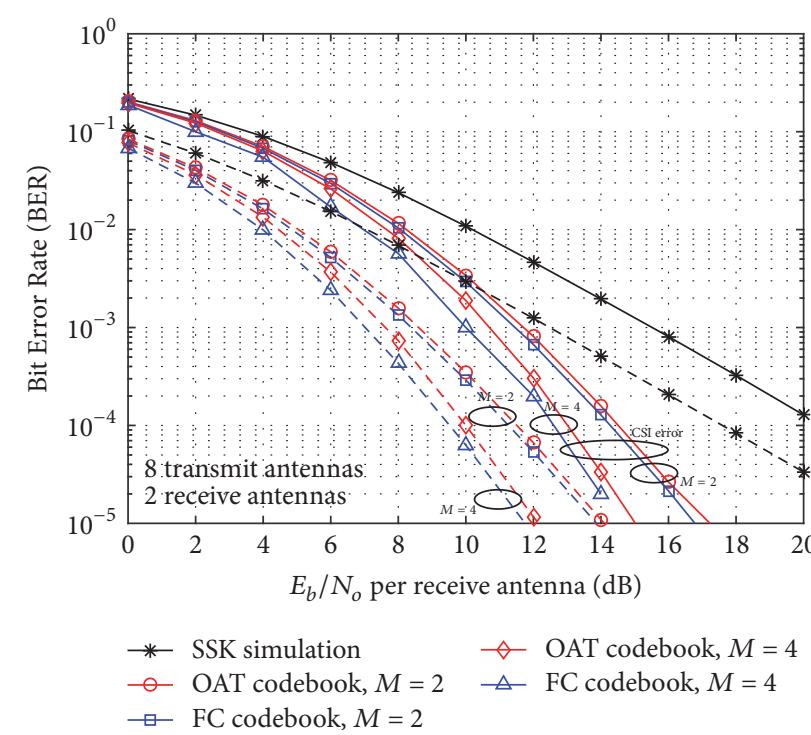

FIGURE 11: BER comparison between perfect and imperfect CSI at the receiver for $N_{t}=8$ and $N_{r}=2$. Solid lines denote imperfect CSI and dashed lines denote perfect CSI.

methods incur a loss of about $3 \mathrm{~dB}$. This loss is consistent with results in the literature as in $[3,17]$. Hence, the proposed codebook precoding methods are not oversensitive to CSI error compared to other methods in the literature.

\section{Conclusions}

In this research, firstly, we introduced new analytical derivation for BER of SSK-MIMO system without precoding. Our results demonstrate that the new BER form is more accurate than the available form in the literature, especially at low SNR and/or a low number of receive antennas. Secondly, we proposed a number of codebook-based phase-rotation precodings (PRP) to maximize the Minimum Euclidean Distance (MED) between the received constellation points. The introduced precoding codebooks have many advantages compared to existing schemes; they have systematic structures that allow codeword index feedback from the receiver to the transmitter and they require less mathematical manipulation during codeword search. Performance of FullCombination (FC) codebook with four phases provided the best SNR gain in comparison to conventional SSK without precoding. However, due to its large size, we introduced alternative codebooks with smaller size to reduce the search complexity, namely, Walsh-Hadamard (WH), Quasi-Orthogonal Sequences (QOS), and Orthogonal Array Testing (OAT). They all provided close performance to the FC codebook. On the other hand, we introduced an iterative search technique to find the best codeword from the FC codebook. Thirdly, we demonstrated by numerical simulation that the proposed methods provide significant performance gain compared to the conventional SSK, and the gain is comparable to optimization-based noncodebook methods. Fourthly, we demonstrated that the proposed codebook-based methods are still effective under nonfavorable situations like transmit antenna correlation and channel estimation error. Finally, we show that the proposed methods provide larger gain in Nakagami- $m$ fading channel with $m=2$.

\section{Conflicts of Interest}

The authors declare that they have no conflicts of interest.

\section{Acknowledgments}

This project was supported by the Deanship of Scientific Research at Prince Sattam Bin Abdulaziz University under Research Project no. 2016/01/6637.

\section{References}

[1] P. Yang, M. D. Renzo, Y. Xiao, S. Li, and L. Hanzo, "Design guidelines for spatial modulation," IEEE Communications Surveys Tutorials, vol. 17, pp. 6-26, May 2015.

[2] M. D. Renzo, H. Haas, A. Ghrayeb, S. Sugiura, and L. Hanzo, "Spatial modulation for generalized MIMO: challenges, opportunities, and implementation," Proceedings of the IEEE, vol. 102, no. 1, pp. 56-103, 2014.

[3] J. Jeganathan, A. Ghrayeb, L. Szczecinski, and A. Ceron, "Space shift keying modulation for MIMO channels," IEEE Transactions on Wireless Communications, vol. 8, no. 7, pp. 3692-3703, 2009.

[4] J. Jeganathan, A. Ghrayeb, and L. Szczecinski, "Generalized space shift keying modulation for MIMO channels," in Proceedings of the 2008 IEEE 19th International Symposium on Personal, Indoor and Mobile Radio Communications, PIMRC 2008, pol, September 2008.

[5] A. Younis, N. Serafimovski, R. Mesleh, and H. Haas, "Generalised spatial modulation," in Proceedings of the 44th Asilomar Conference on Signals, Systems and Computers, Asilomar 2010, pp. 1498-1502, usa, November 2010.

[6] R. Y. Mesleh, H. Haas, S. Sinanović, C. W. Ahn, and S. Yun, "Spatial modulation," IEEE Transactions on Vehicular Technology, vol. 57, no. 4, pp. 2228-2241, 2008.

[7] A. Younis, R. Mesleh, H. Haas, and P. M. Grant, "Reduced complexity sphere decoder for spatial modulation detection receivers," in Proceedings of the 53rd IEEE Global Communications Conference, GLOBECOM 2010, usa, December 2010.

[8] J. Jeganathan, A. Ghrayeb, and L. Szczecinski, "Spatial modulation: Optimal detection and performance analysis," IEEE Communications Letters, vol. 12, no. 8, pp. 545-547, 2008.

[9] P. Yang, Y. Xiao, Y. L. Guan et al., "Single-carrier SM-MIMO: a promising design for broadband large-scale antenna systems," IEEE Communications Surveys \& Tutorials, vol. 18, no. 3, pp. 1687-1716, 2016.

[10] P. Yang, Y. Xiao, Y. Yu, and S. Li, "Adaptive spatial modulation for wireless mimo transmission systems," IEEE Communications Letters, vol. 15, no. 6, pp. 602-604, 2011

[11] M. Maleki, H. R. Bahrami, S. Beygi, M. Kafashan, and N. H. Tran, "Space modulation with CSI: Constellation design and performance evaluation," IEEE Transactions on Vehicular Technology, vol. 62, no. 4, pp. 1623-1634, 2013.

[12] A. Garcia-Rodriguez, C. Masouros, and L. Hanzo, "Pre-scaling optimization for space shift keying based on semidefinite relaxation," IEEE Transactions on Communications, vol. 63, no. 11, pp. 4231-4243, 2015. 
[13] M.-C. Lee, W.-H. Chung, and T.-S. Lee, “Generalized precoder design formulation and iterative algorithm for spatial modulation in MIMO systems with CSIT," IEEE Transactions on Communications, vol. 63, no. 4, pp. 1230-1244, 2015.

[14] M. Di Renzo and H. Haas, "Improving the performance of space shift keying (SSK) modulation via opportunistic power allocation," IEEE Communications Letters, vol. 14, no. 6, pp. 500-502, 2010.

[15] P. Yang, Y. Xiao, B. Zhang, M. El-Hajjar, S. Li, and L. Hanzo, "Phase rotation-based precoding for spatial modulation systems," IET Communications, vol. 9, no. 10, pp. 1315-1323, 2015.

[16] C. Masouros, "Improving the diversity of spatial modulation in MISO channels by phase alignment," IEEE Communications Letters, vol. 18, no. 5, pp. 729-732, 2014.

[17] P. Yang, Y. L. Guan, Y. Xiao, M. Di Renzo, S. Li, and L. Hanzo, "Transmit precoded spatial modulation: maximizing the minimum euclidean distance versus minimizing the bit error ratio," IEEE Transactions on Wireless Communications, vol. 15, no. 3, pp. 2054-2068, 2016.

[18] P. Yang, Y. Xiao, S. Li, and L. Hanzo, "A low-complexity power allocation algorithm for multiple-input-multiple-output spatial modulation systems," IEEE Transactions on Vehicular Technology, vol. 65, no. 3, pp. 1819-1825, 2016.

[19] M. S. Veedu, C. R. Murthy, and L. Hanzo, "Single-RF spatial modulation relying on finite-rate phase-only feedback: Design and analysis," IEEE Transactions on Vehicular Technology, vol. 65, no. 4, pp. 2016-2025, 2016.

[20] P. Yang, Y. Xiao, B. Zhang, S. Li, M. El-Hajjar, and L. Hanzo, "Power allocation-aided spatial modulation for limitedfeedback MIMO systems," IEEE Transactions on Vehicular Technology, vol. 64, no. 5, pp. 2198-2204, 2015.

[21] D. J. Love, R. W. Heath Jr., V. K. N. Lau, D. Gesbert, B. D. Rao, and M. Andrews, "An overview of limited feedback in wireless communication systems," IEEE Journal on Selected Areas in Communications, vol. 26, no. 8, pp. 1341-1365, 2008.

[22] M.-C. Lee, W.-H. Chung, and T.-S. Lee, "Precoder design for space shift keying in MIMO systems with limited feedback," in Proceedings of the 2014 25th IEEE Annual International Symposium on Personal, Indoor, and Mobile Radio Communication, IEEE PIMRC 2014, pp. 6-10, usa, September 2014.

[23] C. Masouros and L. Hanzo, "Constellation randomization achieves transmit diversity for single-rf spatial modulation," IEEE Transactions on Vehicular Technology, vol. 65, no. 10, pp. 8101-8111, 2016.

[24] H. Noh, Y. Kim, J. Lee, and C. Lee, "Codebook design of generalized space shift keying for FDD massive MIMO systems in spatially correlated channels," IEEE Transactions on Vehicular Technology, vol. 64, no. 2, pp. 513-523, 2015.

[25] M. Al-Ansi, S. A. Aljunid, and E. Sourour, "Phase rotation codebook precoding for space shift keying MIMO systems," in Mobile and Wireless Technologies 2017, vol. 425 of Lecture Notes in Electrical Engineering, pp. 94-101, Springer Singapore, Singapore, 2018.

[26] L. Collin, O. Berder, P. Rostaing, and G. Burel, "Optimal minimum distance-based precoder for MIMO spatial multiplexing systems," IEEE Transactions on Signal Processing, vol. 52, no. 3, pp. 617-627, 2004.

[27] M.-C. Lee, W.-H. Chung, and T.-S. Lee, "Limited feedback precoder design for spatial modulation in MIMO systems," IEEE Communications Letters, vol. 19, no. 11, pp. 1909-1912, 2015.

[28] A. Gersho and R. M. Gray, Vector Quantization and Signal Compression, Springer, Boston, MA, USA, 1992.
[29] Y.-H. Lin, Y.-H. Chen, C.-Y. Chu, C.-Z. Zhan, and A.-Y. Wu, "Dual-mode low-complexity codebook searching algorithm and VLSI architecture for LTE/LTE-advanced systems," IEEE Transactions on Signal Processing, vol. 61, no. 14, pp. 3545-3562, 2013.

[30] T. Handte, A. Müller, and J. Speidel, "BER analysis and optimization of generalized spatial modulation in correlated fading channels," in Proceedings of the 2009 IEEE 70th Vehicular Technology Conference Fall, VTC 2009 Fall, pp. 1-5, September 2009.

[31] M. Di Renzo and H. Haas, "Bit error probability of SMMIMO over generalized fading channels," IEEE Transactions on Vehicular Technology, vol. 61, no. 3, pp. 1124-1144, 2012.

[32] J. Proakis and M. Salehi, Digital Communiucations, McHrawHill, New York, NY, USA, November 2007.

[33] M. Simon and M. Alouini, Digital communications over fading channels, a Unified Approach to Performance Analysis, John Wiley Sons, New York, NY, USA, July 2000.

[34] D. L. F. Olver, R. Boisvert, and C. Clark, NIST Handbook of Mathematical Functions, Cambridge University Press, New York, NY, USA, 2010.

[35] L. Wu, H. Yang, and D. Wang, "Hadamard transform based codebook design for uniform circular arrays in mobile radio communications," in Proceedings of the 76th IEEE Vehicular Technology Conference, VTC Fall 2012, can, September 2012.

[36] K. Yang, Y.-K. Kim, and P. V. Kumar, "Quasi-orthogonal sequences for code-division multiple-access systems," Institute of Electrical and Electronics Engineers Transactions on Information Theory, vol. 46, no. 3, pp. 982-993, 2000.

[37] R. W. Heath, T. Strohmer, and A. J. Paulraj, "On quasiorthogonal signatures for CDMA systems," Institute of Electrical and Electronics Engineers Transactions on Information Theory, vol. 52, no. 3, pp. 1217-1226, 2006.

[38] A. S. Hedayat, N. J. A. Sloane, and J. Stufken, Orthogonal Arrays: Theory and Applications, Springer, New York, NY, USA, 1999.

[39] Y. Cai and D. Liu, "Multiuser detection using the taguchi method for DS-CDMA systems," IEEE Transactions on Wireless Communications, vol. 4, no. 4, pp. 1594-1607, 2005.

[40] N. J. A. Sloane, A Library of Orthogonal Arrays, 2005, http://neilsloane.com/oadir/.

[41] W. F. Kuhfeld, "SAS Technical Support Notes," Tech. Rep., 2009, http://support.sas.com/techsup/technote/ts723.html.

[42] W.-C. Weng, F. Yang, and A. Z. Elsherbeni, "Linear antenna array synthesis using Taguchi's method: a novel optimization technique in electromagnetics," IEEE Transactions on Antennas and Propagation, vol. 55, no. 3 I, pp. 723-730, 2007.

[43] W. F. Kuhfeld, A library of Strength-Two Orthogonal arrays, 2009, http://support.sas.com/techsup/technote/ts723_Designs.txt.

[44] N. J. A. Sloane, A Library of Orthogonal Arrays with 64 Runs and 21 Factors, 2005, http://neilsloane.com/oadir/oa.64.21.4.2.txt.

[45] N. J. A. Sloane, A Library of Orthogonal Arrays with 32 Runs and 9 Fcators, 2005, http://neilsloane.com/oadir/oa.32.9.4.2.txt. 


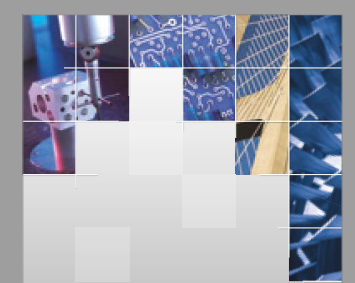

\section{Enfincering}
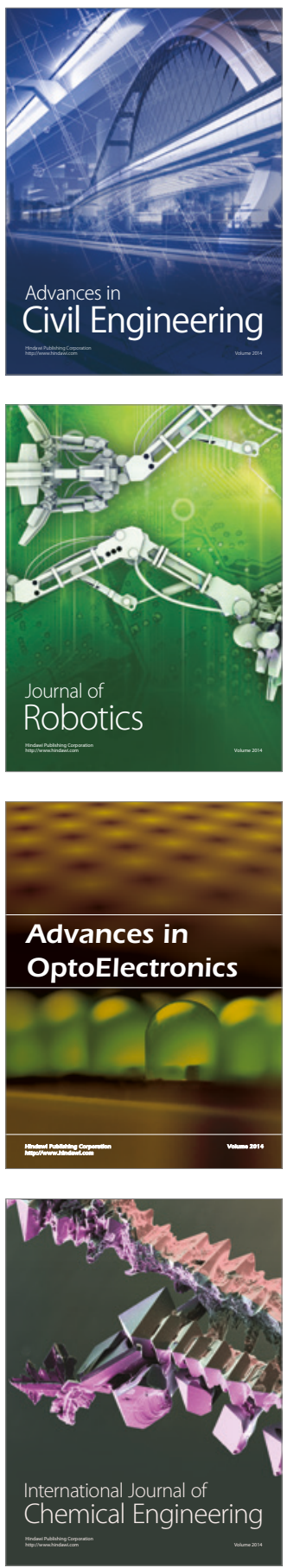

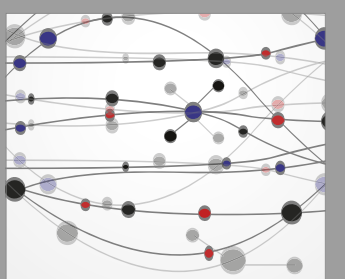

The Scientific World Journal

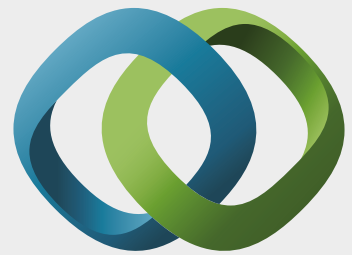

\section{Hindawi}

Submit your manuscripts at

https://www.hindawi.com
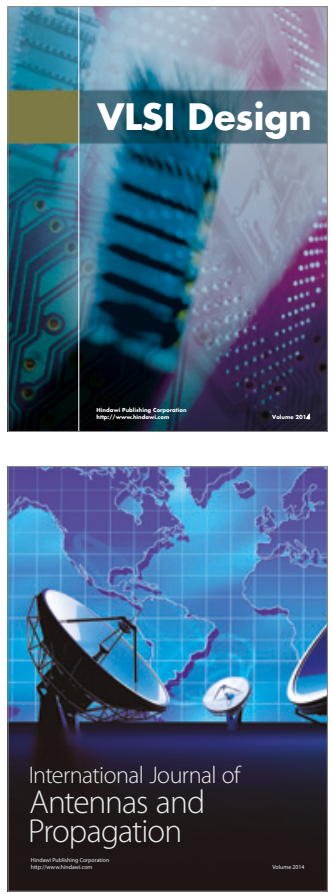

\section{Rotating}

Machinery
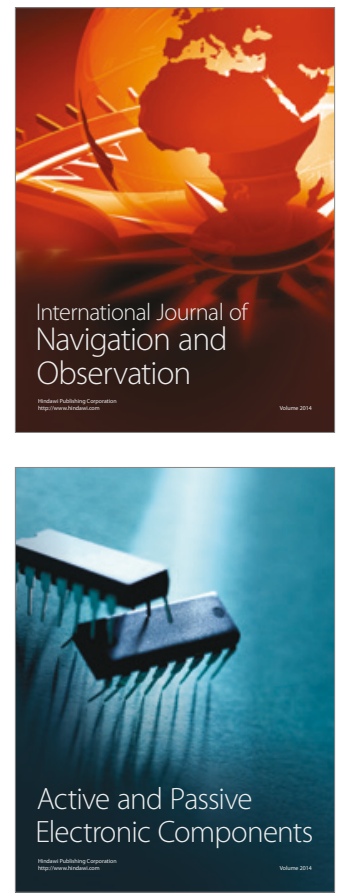
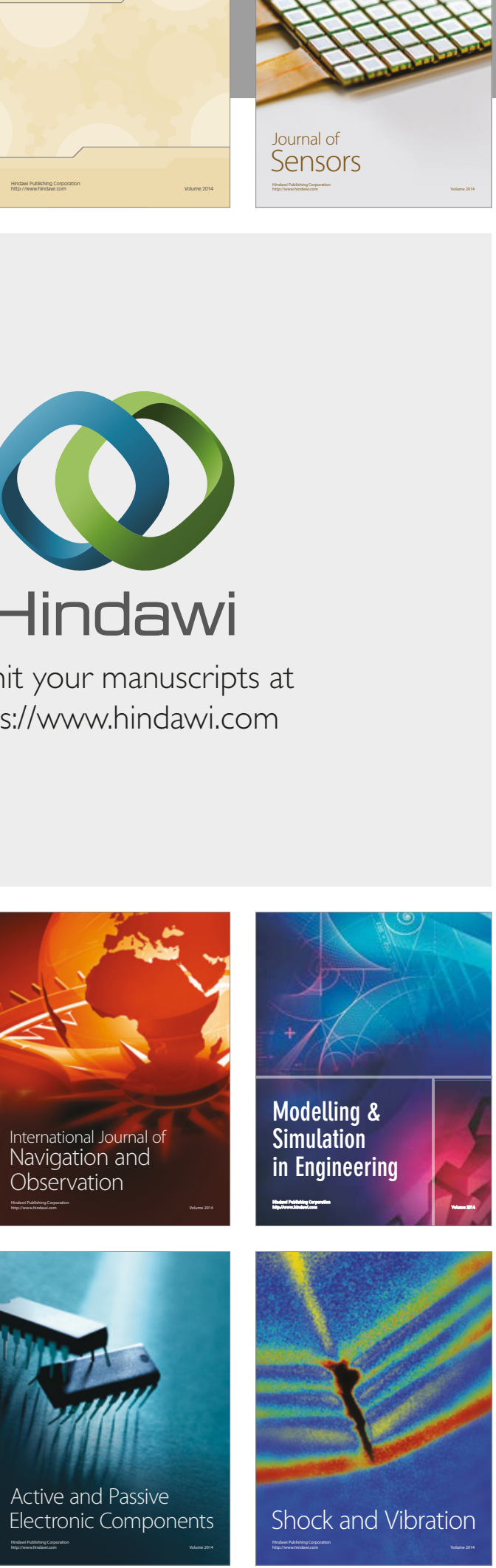
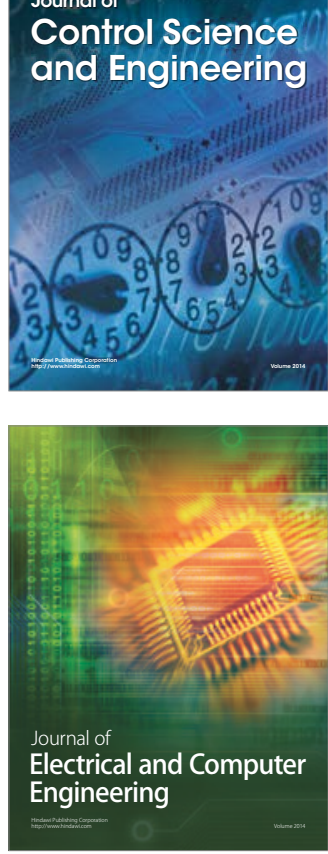

Distributed

Journal of

Control Science

and Engineering
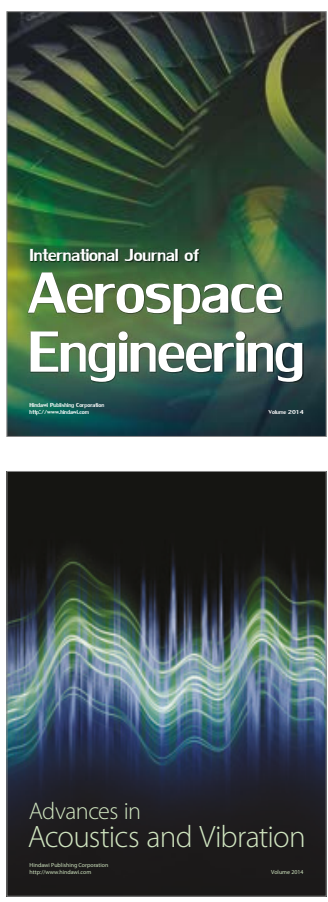

Sensor Networks 